\title{
STUDIES ON IMMUNITY IN ANTHRAX
}

\section{Factors Influencing Elaboration of the Protective Antigen of Bacillus anthracis in Chemically Defined Media}

\author{
MILTON PUZISS ${ }^{1}$ AND GEORGE G. WRIGHT \\ Chemical Corps Biological Laboratories, Camp Detrick, Frederick, Maryland
}

Received for publication April 19, 1954

Elaboration of the protective antigen of Bacillus anthracis in a chemically defined, nonprotein medium was described in a previous report from these laboratories (Wright et al., 1954). This medium, referred to as 528 medium, was composed of 17 amino acids, glutamine, adenine, guanine, uracil, thiamin, glucose, sodium bicarbonate, and inorganic salts. Sterile filtrates of cultures of $B$. anthracis in the medium produced effective immunity in rabbits, guinea pigs, and monkeys; the dose required to induce significant immunity in rabbits was of the same order as that of the edema fluid of anthrax lesions (Cromartie et al., 1947). Certain minor modifications in the composition of the medium markedly influenced the antigenic potency, suggesting that elaboration of antigen is associated with a particular type of metabolic activity of the organism.

Further knowledge of the conditions for elaboration of antigen was desirable to facilitate production of antigen on a practical scale, to indicate the general relationship of antigen elaboration to growth of the organism, and to provide information desirable for investigating the existence and significance of extracellular antigens of this general type in other organisms. Certain relationships of constituents of the medium and conditions of growth to accumulation of protective antigen were therefore investigated in greater detail; considerable simplification and improvement of the medium and cultural conditions were found to be possible. The results suggest certain preliminary inferences regarding the metabolic processes associated with elaboration of antigen.

\section{MATERIALS AND METHODS}

Cultures. A standard spore suspension of the Vollum strain of $B$. anthracis was used

${ }^{1}$ Present address : Department of Bacteriology, University of Southern California, Los Angeles, California. throughout for challenge of animals in the assay procedure as described previously (Wright and Slein, 1951). Strain NP-A, a nonproteolytic mutant of the Vollum strain that had been employed in previous studies (Wright et al., 1954), was used as the inoculum for production of antigen during the early part of the work. During the later studies strain R1-NP, a nonencapsulated and nonproteolytic double mutant, was substituted. This strain was obtained from the Vollum strain by isolation of a nonencapsulated mutant by the method of Sterne (1937), followed by selection of a nonproteolytic mutant as described previously (Wright et al., 1951). The strain was avirulent for guinea pigs in doses of 1,000 spores.

Reagents. All chemicals were of reagent or equivalent grade and were obtained from commercial sources. Water redistilled in glass was used in the preparation of media. Synthetic citrovorum factor was supplied by Dr. H. P. Broquist of Lederle Laboratories Division, American Cyanamid Company.

Preparation of protective antigen. In most experiments the test media were prepared in $250 \mathrm{ml}$ volumes in Fernbach flasks stoppered with cotton plugs as described previously (Wright et al., 1954). The media were autoclaved for 15 minutes at $120 \mathrm{C}$; longer autoclaving led to less satisfactory growth. The media were seeded with an inoculum of 500 spores per $\mathrm{ml}$ of medium and incubated statically at $37 \mathrm{C}$. After growth the cultures were adjusted to a $\mathrm{pH}$ of approximately 8 by addition of $\mathrm{N}$ sodium hydroxide, and the antigen containing filtrates were obtained by passage through sintered glass filters of ultrafine porosity. Cultures of the NP-A strain tended to clog the filters, and it was necessary to remove most of the organisms by prior centrifugation at about $1,500 \mathrm{rpm}$. Small disc type filters were used in the early work; candle type filters, in the later work. The latter had a larger area and allowed more 
rapid filtration. Culture filtrates were dried by the lyophil method and stored over calcium chloride at room temperature. Experience indicated that deterioration of properly dried preparations was very slow under these conditions. The yield was approximately $5 \mathrm{mg}$ of dried material per $\mathrm{ml}$ of culture filtrate. Lyophilized preparations were hygroscopic, and their antigenic activity deteriorated in the presence of moisture.

Assay of protective antigen. Protective antigen activity was estimated by immunization and challenge of guinea pigs since previous work had indicated that these animals could be immunized readily by filtrates of cultures grown in chemically defined media (Wright et al., 1954). The lyophilized filtrates were dissolved at the desired concentration in distilled water and stored at $-20 \mathrm{C}$ in rubber-stoppered tubes until the time of injection. Albino guinea pigs, weighing $250-300 \mathrm{~g}$, were injected subcutaneously on the left flank with a total of $2.5 \mathrm{ml}$ of the test preparation in five doses given at 2 day intervals. At least three animals were injected with each preparation. Seven days after the last immunizing injection, the animals and a group of unimmunized controls were challenged intracutaneously on the right flank by injection of 1,000 spores of the Vollum strain and observed for 14 days. This challenge dose represented approximately $200 \mathrm{LD}_{50}$ and was invariably fatal for the control animals, usually within 4 days.

\section{EXPERIMENTAL RESULTS}

Amino acid requirements. The 528 medium for production of protective antigen described in the previous report (Wright et al., 1954) contained 17 amino acids and glutamine in the concentrations given in table 1 . The relationship of these constituents to growth of the organism and elaboration of protective antigen was studied by omitting them individually from the medium. The 528 reference medium was set up in parallel with each group of modified media. Growth was estimated by visual observation, and elaboration of antigen by assay of the filtrates in guinea pigs. The lyophilized filtrates were dissolved for assay at a concentration of $25 \mathrm{mg}$ per ml, which represented a 5 -fold concentration of the original filtrate. Thus, each animal received a total amount equivalent to $12.5 \mathrm{ml}$ of the original filtrate. This dose was sufficient to protect nearly all guinea pigs when filtrates from the reference medium were used. Significantly less than complete survival at this level was considered evidence of inhibition of antigen production. Results of the experiments are summarized in table 2 .

It may be noted that individual omission of 11 of the amino acids had no apparent influence on growth of the organism; the cultures were accordingly filtered and assayed for protective antigen after the incubation time of approximately 24 hours that had been satisfactory for the reference medium. Omission of glycine, alanine, lysine, cystine, or glutamine appeared to have no significant effect on elaboration of antigen, whereas omission of isoleucine, aspartic acid, glutamic acid, arginine, methionine, or tryptophan inhibited antigen elaboration to an appreciable extent.

Omission of five amino acids resulted in only slight growth after 24 hours' incubation but essentially normal growth after 45-48 hours; the cultures were assayed for protective antigen after this period. Of the five only threonine was not essential for antigen production; omission of leucine, histidine, proline, or phenylalanine produced inhibition of antigen elaboration as well as delay in growth. Omission of serine or valine led to almost complete inhibition of growth, and accordingly no test was made for elaboration of antigen.

Reduction of the concentration of threonine to $0.0005 \mathrm{M}$ or of proline to $0.00025 \mathrm{M}$ (one-half and one-quarter, respectively, of their concentrations in the reference medium) did not influence growth or elaboration of antigen. These modifications were of practical importance since threonine and proline were the most expensive constituents of the medium. It was found that cystine could be replaced by cysteine hydrochloride, which is more readily soluble. Growth and antigen elaboration were unchanged when equimolar concentrations of asparagine or glutamine were substituted, respectively, for aspartic or glutamic acid. No detectable antigen was elaborated when aspartic acid was replaced by equimolar oxalacetic, fumaric, or malic acids. Twofold increase in the concentration of glutamic acid to $0.0022 \mathrm{M}$ replaced the requirement for proline for antigen elaboration but did not allow growth at the normal rate. Threefold increase in the concentration of aspartic acid to $0.0015 \mathrm{~m}$ did not replace threonine as a growth stimulatory factor.

Combinations of the omissions and alterations 
TABLE 1

Composition of reference media for elaboration of protective antigen

\begin{tabular}{|c|c|c|c|c|}
\hline \multirow{2}{*}{ Constituent } & \multicolumn{4}{|c|}{ Molar Concentration } \\
\hline & 528 Medium & 555 Medium & 599 Medium & 687 Medium \\
\hline Glycine. & 0.0004 & - & - & - \\
\hline DL-Alanine $\ldots \ldots \ldots \ldots \ldots \ldots \ldots$ & 0.001 & - & - & - \\
\hline DL-Serine..$\ldots \ldots \ldots \ldots \ldots \ldots$ & 0.0002 & 0.0002 & 0.0002 & 0.0002 \\
\hline DL-Threonine.......... & 0.001 & 0.0005 & 0.0005 & 0.0005 \\
\hline DL-Valine.............. & 0.0005 & 0.0005 & 0.0005 & 0.0005 \\
\hline DL-Leucine & 0.001 & 0.001 & 0.001 & 0.001 \\
\hline DL-Isoleucine ............ & 0.001 & 0.001 & 0.001 & 0.001 \\
\hline DL-Aspartic acid............... & 0.0005 & 0.0005 & 0.0005 & 0.0005 \\
\hline L-Glutamic acid $\ldots \ldots \ldots \ldots \ldots \ldots$ & 0.0011 & 0.0011 & 0.0011 & 0.0011 \\
\hline DL-Lysine $\cdot \mathrm{HCl} \ldots \ldots \ldots \ldots$ & 0.00016 & - & - & - \\
\hline $\mathrm{L}$-Arginine $\cdot \mathrm{HCl}$ & 0.0005 & 0.0005 & 0.0005 & 0.0005 \\
\hline $\mathrm{L}-\mathrm{Histidine} \cdot \mathrm{HCl}$. & 0.0005 & 0.0005 & 0.0005 & 0.0005 \\
\hline L-Cystine $\ldots \ldots \ldots \ldots \ldots$ & 0.00005 & - & 一 & - \\
\hline L-Cysteine $\cdot \mathrm{HCl} \ldots \ldots \ldots \ldots$ & - & 0.00005 & 0.00005 & - \\
\hline DL-Methionine $\ldots \ldots \ldots \ldots$ & 0.0002 & 0.0002 & 0.0002 & 0.0002 \\
\hline L-Proline $\ldots \ldots \ldots \ldots \ldots$ & 0.001 & 0.00025 & 0.00025 & 0.00025 \\
\hline DL-Phenylalanine.......... & 0.0004 & 0.0004 & 0.0004 & 0.0004 \\
\hline L-Tryptophan. . & 0.00025 & 0.00025 & 0.00025 & 0.00025 \\
\hline L-Glutamine. . . & $* 0.000007$ & - & - & - \\
\hline Calcium chloride $\ldots \ldots \ldots \ldots \ldots \ldots$ & 0.0001 & 0.0001 & 0.0001 & 0.0001 \\
\hline Magnesium sulfate................ & 0.00004 & 0.00004 & 0.00004 & 0.00004 \\
\hline Manganous sulfate......... & $* 0.000005$ & $* 0.000005$ & 0.000005 & 0.000005 \\
\hline Ferrous sulfate $\ldots \ldots \ldots \ldots \ldots \ldots$ & $* 0.0005$ & $* 0.0005$ & $*_{0.0005}$ & 0.00005 \\
\hline Potassium dihydrogen phosphate.. & 0.005 & 0.005 & 0.005 & 0.005 \\
\hline Dipotassium hydrogen phosphate.. & 0.005 & 0.005 & 0.005 & 0.005 \\
\hline Sodium bicarbonate.......... & $* 0.03$ & $* 0.03$ & $* 0.03$ & ${ }^{*} 0.03$ \\
\hline Adenine.... & 0.00002 & 0.00002 & - & - \\
\hline Guanine $\cdot \mathbf{H C l} \ldots \ldots \ldots \ldots$ & 0.00002 & 0.000014 & 0.000014 & - \\
\hline Uracil.............. & 0.000025 & 0.000025 & - & - \\
\hline Xanthine $\ldots \ldots \ldots \ldots \ldots$ & - & - & - & 0.00004 \\
\hline Thiamin $\cdot \mathrm{HCl}$. & 0.0000003 & 0.0000003 & 0.0000003 & 0.0000003 \\
\hline Glucose $\ldots \ldots \ldots \ldots$ & $* 0.0056$ & $* 0.0056$ & $* 0.0056$ & 0.0056 \\
\hline
\end{tabular}

* These constituents were sterilized separately and added aseptically to the autoclaved media containing the other constituents. Glutamine, manganous sulfate, and ferrous sulfate solutions were sterilized by filtration; sodium bicarbonate and glucose solutions, by autoclaving.

that had been found to be permissible individually were then investigated. The following simultaneous alterations were found to be satisfactory: omission of glycine, alanine, lysine, and glutamine, substitution of equimolar cysteine hydrochloride for cystine, and reduction in the concentration of threonine to one-half and of proline to onefourth that in the 528 medium. Cystine was replaced by cysteine rather than omitted because there was evidence that elaboration of antigen was stimulated slightly by cystine or cysteine. This modified formula, designated 555 medium, was equivalent to 528 medium for antigen production and was used as a new reference medium in further experiments. The composition is given in table 1.

The influence of bicarbonate and functionally related compounds. Gladstone (1946) demonstrated that bicarbonate was an essential factor for elaboration of protective antigen in complex media. In addition, bicarbonate was shown to be necessary for production of the capsular polypeptide by virulent strains of $B$. anthracis (Thorne et al., 1953). The requirement for bicarbonate in 528 medium was therefore investigated. Although the organism grew at a 
TABLE 2

Effect of omission of amino acids on growth and antigen elaboration by the NP-A strain in 528 medium

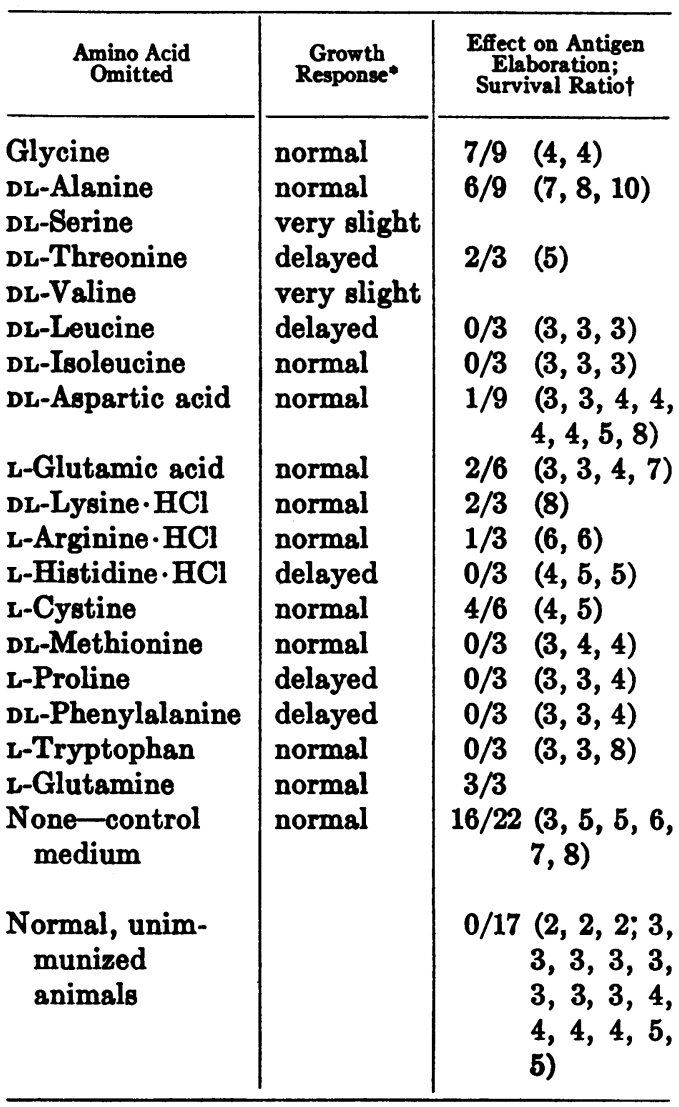

* Growth estimated visually as equivalent to the control medium after 24 hours was termed normal. Growth less than in the control medium at 24 hours but essentially equivalent after 48 hours' incubation was termed delayed.

† Survival ratios are recorded as the number of animals surviving divided by the number challenged. Numbers in parentheses refer to the day after challenge on which deaths occurred.

normal rate when bicarbonate was omitted, or when the bicarbonate-free medium was aerated with carbon dioxide-free air, no detectable antigen was elaborated.

When bicarbonate was omitted, the cultures became acid during growth, and it seemed possible that buffering activity represented the essential role of the bicarbonate. Accordingly, cultures were grown in bicarbonate-free medium and maintained at the same $\mathrm{pH}$ as the bicarbo- nate containing control by periodic addition of sterile $\mathrm{NaOH}$. No protective antigen could be detected in filtrates of these cultures. Reduction in the concentration of bicarbonate to one-half $(0.015 \mathrm{M})$ that of the control medium resulted in a marked decrease in antigen elaboration, and reduction to one-fourth $(0.0075 \mathrm{M})$ the normal concentration or below prevented elaboration of detectable antigen.

Attempts were made to replace bicarbonate by compounds known to be involved in carbon dioxide metabolism of other organisms. No detectable antigen was produced when bicarbonate was replaced by $0.004 \mathrm{M}$ citrate, succinate, fumarate, or malate. In addition, no significant stimulation of antigen elaboration was observed when these compounds were added to media containing bicarbonate; addition of $0.001 \mathrm{M}$ sodium citrate to the control medium caused a marked inhibition of antigen elaboration, perhaps because of complex formation with the metal ions in the medium. Synthetic citrovorum factor and folic acid were added individually to the medium at a concentration of $0.1 \mu \mathrm{g} / \mathrm{ml}$. In a bicarbonate-free medium maintained at the same $\mathrm{pH}$ as the bicarbonate containing control, they did not stimulate the elaboration of appreciable protective antigen. When added to the bicarbonate containing medium, they did not increase the elaboration of antigen. Tenfold increase in the concentration of thiamin had no appreciable effect on growth or on accumulation of antigen.

Requirements for metal ions. Omission of calcium chloride from the medium did not affect growth significantly but caused a marked reduction in elaboration of protective antigen. Omission of magnesium sulfate resulted in almost complete inhibition of growth, in agreement with previous observations (Brewer et al., 1946). Doubling the concentrations of calcium and magnesium salts did not increase the elaboration of antigen. Omission of manganous sulfate had no apparent effect either on growth or on elaboration of antigen. Probably the ferrous sulfate, present in the basal medium at a concentration of $0.0005 \mathrm{M}$, supplied sufficient manganese for normal metabolism. Elimination of added manganous sulfate did not seem desirable, however, since any subsequent reduction in the concentration of ferrous sulfate might then result in manganese deficiency. Satisfactory 
results were obtained when the manganous sulfate was autoclaved with the other constituents of the medium.

The 528 and 555 media contained $0.0005 \mathrm{M}$ ferrous sulfate which was sterilized by filtration and added aseptically to the autoclaved media. A definite opalescence appeared when the solution was added, and a precipitate, presumably of ferrous phosphate, settled out of the media during incubation. Growth was very slow when ferrous sulfate was omitted. Reduction in the concentration of ferrous sulfate to $0.00005 \mathrm{M}$ or to $\mathbf{0 . 0 0 0 0 1 7} \mathrm{M}$ resulted in a delay in growth, but after 45 hours' incubation good growth and normal antigen elaboration were obtained. Autoclaving the ferrous sulfate with the other components of the medium caused a delay in growth, but moderate antigen production was detected after the longer incubation period. Apparently, ferrous sulfate functioned as a growth stimulatory substance, and this property was not retained when the salt was autoclaved with the other constituents. Ferric sulfate autoclaved with the medium replaced ferrous sulfate for elaboration of antigen in concentrations as low as $0.000017 \mathrm{M}$, but approximately 48 hours were required for normal growth. No stimulation of growth was noted when amounts of cobalt, copper, zinc, or lithium salts ranging from $10^{-1}$ to $10^{-7} \mathrm{M}$ were added to the medium containing $1 / 10$ the normal concentration of ferrous sulfate, indicating that the growth stimulatory activity of ferrous sulfate was not the result of trace metal contaminants.

Substitution of a nonencapsulated strain for production of antigen. Gladstone (1946) had shown that the Weybridge strain of $B$. anthracis, an avirulent, nonencapsulated mutant, was capable of producing protective antigen during cultivation in vitro. Preliminary experiments indicated that strain R1-NP, a nonencapsulated, nonproteolytic double mutant derived from the Vollum strain, produced amounts of protective antigen at least equivalent to the NP-A strain when cultivated in 555 medium. Use of the R1-NP strain was desirable from a practical standpoint since the absence of capsular material allowed the cultures to be filtered readily without prior centrifugation. Furthermore, the danger of manipulating large volumes of virulent anthrax culture and the potential hazard involved in practical use of filtrates derived from such cultures were also avoided. Accordingly, a spore suspension of the R1-NP strain was used as inoculum in the subsequent work, except as indicated.

Requirement for purines and pyrimidines. The effect of purines, pyrimidines, and related compounds on growth and elaboration of antigen was studied in the 555 reference medium. This medium contained $0.00002 \mathrm{M}$ adenine and uracil and $0.000014 \mathrm{M}$ guanine. The lower concentration of guanine was carried over from earlier work in which the hydrochloride had been used without appropriate correction. Initial experiments were carried out with the NP-A strain. Omission of all purines and pyrimidines simultaneously caused a delay in growth and a considerable reduction in elaboration of antigen, even when the incubation time was increased to allow equivalent growth. Although the NP-A strain could readily be adapted to grow at the normal rate in the absence of purines or pyrimidines, preliminary results indicated that no protective antigen was elaborated by the adapted strain under these conditions. When the three constituents were tested individually and in combinations, only guanine stimulated growth and production of antigen at the normal rate.

Further studies of various purine and pyrimidine compounds were carried out with the R1-NP strain. The compounds at a concentration of $0.000014 \mathrm{M}$ were added individually to $\mathbf{5 5 5}$ medium from which adenine, guanine, and uracil were omitted. It was found that guanosine, adenosine, xanthine, or hypoxanthine was essentially equivalent to guanine in supporting growth and elaboration of antigen. Guanylic acid or 5-adenylic acid was apparently somewhat less effective than guanine in both respects. Uric acid, cytidine sulfate, cytosine, thymine, or uridine produced no appreciable stimulation of growth nor antigen elaboration. Addition of 0.000014 M 8-chloroxanthine or benzimidazole had no effect on growth or antigen production, either in the purine-free medium or in the presence of equimolar guanine.

Normal growth and satisfactory elaboration of antigen were obtained when the concentration of guanine, guanosine, adenosine, or xanthine was increased 10 -fold to $0.00014 \mathrm{~m}$. This concentration of adenine, however, inhibited growth and antigen elaboration, both in the purine-free medium and in the presence of $0.000014 \mathrm{M}$ guanine. The 
inhibitory effect of a high concentration of adenine also had been noted in preliminary work with the NP-A strain. It was concluded that either guanine or xanthine at a concentration between $0.000014 \mathrm{M}$ and $0.00014 \mathrm{M}$ was a suitable purine supplement for antigen production.

The 555 medium was further simplified by omission of adenine and uracil, and by autoclaving the manganous sulfate with the medium. This combination of modifications was referred to as 599 medium (table 1). The basic medium was prepared by 25-fold dilution of two stock solutions, one containing the phosphate salts and the other containing all other constituents except ferrous sulfate, glucose, and sodium bicarbonate. Solutions of the latter constituents were sterilized separately and added aseptically to the autoclaved medium. The medium supported normal growth, and elaboration of protective antigen in several 5 to 10 liter lots was equivalent to that obtained in 528 medium.

Amino acid requirements of the R1-NP strain. Preliminary experiments suggested that the amino acid requirements of the R1-NP strain in 599 medium were somewhat different from those determined in the initial experiments. Certain amino acid requirements were therefore reinvestigated; typical results are presented in table 3. In most respects the results were in agreement with those obtained previously. The requirements for leucine, isoleucine, methionine, proline, phenylalanine, and histidine were confirmed. However, considerable elaboration of antigen occurred in the absence of aspartic acid, and normal elaboration of antigen occurred in the absence of glutamic acid if the incubation time was increased to 48 hours to overcome the delay in growth. Proline was not required for normal growth although it remained necessary for elaboration of antigen. The requirements for arginine and tryptophan were not clear-cut, but it was concluded that both probably atimulated elaboration of antigen to a moderate extent.

Replacement of methionine by related compounds was investigated. Choline and betaine, known to be donors of labile methyl-groups in biological systems, did not replace methionine for antigen elaboration. Methionine could be replaced by equimolar homocysteine or homocystine although a slight lag in growth occurred when the latter was substituted. Choline plus
TABLE 3

Effect of omission of amino acids on antigen elaboration by the R1-NP strain in 599 medium

\begin{tabular}{|c|c|c|}
\hline Amino Acid Omitted & $\underset{\text { Trowth }}{\text { Time }}$ & $\begin{array}{l}\text { Effect on Antigen } \\
\text { Elaboration; } \\
\text { Survival Ratio* }\end{array}$ \\
\hline & hours & \\
\hline DL-Leucine........ & 48 & $0 / 3(3,3,4)$ \\
\hline DL-Isoleucine..... & 48 & $0 / 3(3,3,8)$ \\
\hline DL-Aspartic acid. & 24 & $2 / 3(7)$ \\
\hline L-Glutamic acid ... & 48 & $3 / 3$ \\
\hline DL-Methionine . . . . . . & 24 & $0 / 3(4,4,7)$ \\
\hline L-Proline $\ldots \ldots \ldots \ldots \ldots$ & 24 & $0 / 3(3,5,6)$ \\
\hline DL-Phenylalanine $\ldots \ldots \ldots$ & 48 & $0 / 3(3,3,4)$ \\
\hline None-control medium.. & 24 & $4 / 4$ \\
\hline L-Arginine $\cdot \mathbf{H C l}$ & 23 & $1 / 3(6,8)$ \\
\hline None-control medium.. & 23 & $4 / 4$ \\
\hline L-Tryptophan .. & 25 & $2 / 3(3)$ \\
\hline L-Histidine $\cdot \mathbf{H C l}$. & 44 & $1 / 3(3,4)$ \\
\hline None-control medium.. & 25 & $2 / 4(5,6)$ \\
\hline $\begin{array}{l}\text { Normal, unimmunized } \\
\text { animals............. }\end{array}$ & & $\begin{array}{c}0 / 9(3,3,3,3,3 \\
3,3,3,4)\end{array}$ \\
\hline
\end{tabular}

* Survival ratios are recorded as the number of animals surviving divided by the number challenged. Numbers in parentheses refer to the day after challenge on which deaths occurred. Antigen preparations were injected at a concentration of $25 \mathrm{mg}$ per $\mathrm{ml}$.

homocysteine in methionine-free medium produced no increase in antigen elaboration over homocysteine alone. Substitution of equimolar ethionine for methionine produced almost complete inhibition of growth.

Further simplification of the medium and cultural conditions. The effect of incubation time on accumulation of protective antigen was studied in $250 \mathrm{ml}$ and in $500 \mathrm{ml}$ volumes of 599 medium in Fernbach flasks. Antigenic potency was estimated at 12 to 15 hour intervals between 23 and 72 hours of incubation. The volume of culture appeared to have no significant effect on elaboration of antigen. Maximum accumulation of antigen occurred between 34 and 46 hours of incubation, and a small decrease in immunizing potency occurred between 46 and 58 hours. In general the influence of incubation time was not marked between 23 and 58 hours, and appreciable 
activity remained after 72 hours. Autolysis became evident after 48 hours of incubation, and after 58 hours numerous degenerate cell forms occurred. The $\mathrm{pH}$ was 7.6 after 34 hours and then increased steadily to approximately $\mathrm{pH} 9.0$ after 58 hours.

Preliminary attempts were made to obtain elaboration of antigen with the NP-A strain in deep layers of 528 medium, by aeration of the culture during growth. Sintered glass spargers were used to aerate $500 \mathrm{ml}$ volumes of medium in glass cylinders. Heavy growth was obtained within 22 hours, and the pink pigment noted previously (Wright et al., 1954) was elaborated. Assays for antigenic activity revealed that only very slight accumulation of antigen occurred. It is possible that better antigen production would be obtained by aeration with a mixture of carbon dioxide and air, as was the case in elaboration of the capsular polypeptide (Thorne et al., 1953).

As the concentration of glucose in the medium was increased, the $\mathrm{pH}$ of the culture after growth for 72 hours became more acid. The 0.1 per cent optimum for production of antigen may result from the fact that this concentration provided sufficient carbohydrate for rapid growth but did not lead to development of excessive acidity. Addition to the medium of an additional 0.1 per cent of glucose after 24 hours' incubation served to maintain the $\mathrm{pH}$ at a nearly constant level of 7.8 to 8.1. The influence of this addition on elaboration of antigen hasnot been determined. It was thought that substitution of maltose for glucose would lead to a more gradual utilization of carbohydrate and minimize the early accumulation of acid. It was found that although maltose supported elaboration of antigen in the absence of glucose, the substitution appeared to offer no significant advantage.

The effects of increased concentrations of guanine and of substitution of xanthine for guanine, were then reinvestigated. Although the differences were minor, it appeared that slightly increased elaboration of antigen occurred when $0.00004 \mathrm{M}$ xanthine was substituted for guanine, and this modification was used subsequently.

Autoclaving of the glucose with the other constituents of the medium had resulted in inhibition of antigen production. It seemed probable that glucose reacted with some constit- uent of the medium during autoclaving, and results with other organisms (Lankford et al., 1947) suggested that cysteine might be involved. Accordingly, the requirement for cysteine was reexamined since the evidence that it was necessary had been somewhat inconclusive. It was found that with the 599 medium and the R1-NP strain, cysteine could be omitted without detectable inhibition of antigen production, and that under these conditions glucose could be autoclaved with the other constituents of the medium. Reinvestigation of the effect of the ferrous sulfate concentration then revealed that when cysteine was omitted and glucose autoclaved with the other constituents, the concentration of ferrous sulfate could be reduced 10 -fold to $0.00005 \mathrm{M}$ or below without inhibition of growth or of antigen production. These results suggest that the relatively high concentration of ferrous sulfate previously necessary for prompt growth had acted to overcome an inhibitory factor that did not appear when glucose was present during autoclaving of the medium. Under these conditions, the ferrous sulfate could be autoclaved with the medium without inhibition of antigen elaboration and without precipitation of ferrous salts.

The final modification of the medium, referred to as 687 medium, had the composition given in table 1. All components except sodium bicarbonate were autoclaved together, and the bicarbonate autoclaved as a 12.5 per cent solution was added aseptically. The pH of the medium was approximately 7.6. An inoculum of 500 spores of the R1-NP strain per ml of medium was added, and the cultures were incubated for approximately 40 hours at $37 \mathrm{C}$. After this period, the $\mathrm{pH}$ had returned to approximately 7.6, and the cultures were adjusted to $\mathrm{pH} 8$ before filtration. The antigenic potencies apparently approached those obtained in the previous media although the results with different preparations were less consistent. Further investigation of the factors involved is in progress.

\section{DISCUSSION}

The experimental results have provided considerable empirical information regarding the conditions for elaboration of protective antigen and have allowed appreciable simplification and improvement of the medium and cultural conditions for production of the antigen. Progress 
in understanding the fundamental metabolic mechanisms associated with elaboration of antigen has been more difficult. Basic difficulties may contribute to this, in addition to the burdensome and rather inaccurate assay procedure for the antigen. Thus, although the term "elaboration of antigen" has been used to refer to the processes leading to production of an active culture filtrate, it is recognized that the potency of filtrates may be determined not only by the rate of elaboration of antigen by the bacteria, but also by such factors as the rate at which antigen is released into the medium, its rate of destruction in the culture, the filterability and stability of antigen in the filtrate, and the possible presence of substances that enhance or diminish antigenicity in the assay animal. In addition, certain of the cultural conditions shown to be essential for elaboration of antigen may be the result of arbitrarily selected experimental conditions, rather than a reflection of basic metabolic mechanisms responsible for elaboration of antigen. It has been observed that elimination or modification of one constituent of the medium may alter the requirement for another constituent.

Subject to these limitations, certain of the observations suggest promising lines of approach to an understanding of the more fundamental aspects of antigen elaboration. Of particular interest are those constituents of the medium that are unnecessary for satisfactory growth of the organism but apparently play a significant role in antigen elaboration. These include bicarbonate, calcium ion, leucine, isoleucine, histidine, methionine, proline, and phenylalanine. Aspartic acid, though not a critical requirement, appears to stimulate elaboration of antigen significantly.

The requirement for bicarbonate, which had been demonstrated previously in complex media (Gladstone, 1946), is perhaps the most interesting of the critical conditions for elaboration of antigen. The failure of attempts to replace bicarbonate by functionally related compounds, or by control of the $\mathrm{pH}$ of the culture, appears to eliminate certain possible explanations of its role in elaboration of antigen. Further experiments with additional intermediates of carbon dioxide utilization will be necessary to complete this line of investigation. It may be significant that the concentration of bicarbonate necessary for elaboration of antigen is relatively high as compared with the other constituents of the medium. This observation, and the failure thus far to replace bicarbonate by other compounds, would be explained if bicarbonate functioned not as a source for carbon dioxide utilization but rather as an inhibitor of a decarboxylation reaction.

Present information does not permit satisfactory interpretation of the amino acid requirements. There would appear to be no basis for deciding whether the critical constituents represent precursors of the antigen, or whether they influence antigen elaboration in some more subtle manner. It is of interest that isoleucine and phenylalanine are also necessary for production of capsular polypeptide by $B$. anthracis although these amino acids have not been detected as constituents of the polypeptide itself (Thorne et al., 1953). Production of polypeptide and elaboration of protective antigen are also analogous in that omission of glutamic acid delays but does not reduce the accumulation of both substances. Successful replacement of methionine by homocysteine or homocystine, but not by choline or betaine, would suggest that activity as a methyl donor does not represent the crucial function of methionine in elaboration of antigen.

Purines or their derivatives would not appear to be directly associated with elaboration of antigen but rather to affect the process through stimulation of growth of the organism. Thus, elaboration of antigen was detected in the absence of purines if the incubation time was lengthened until good growth was obtained, and the ability of various purine compounds to enhance production of antigen was apparently correlated with their ability to stimulate growth. Among the amino acids, threonine was apparently in a similar category.

Exploratory experiments have indicated that 555 medium does not support satisfactory growth of all strains of $B$. anthracis, presumably because it has been adjusted to the specific nutritional requirements of mutants of the Vollum strain. Further work will be required to determine the extent and basis of this specificity. The present data are also inadequate to establish whether the differences in amino acid requirements noted in the initial and later experiments reflected differences between the NP-A and 
R1-NP strains, or whether they were the result of intervenient alterations in the composition of the medium.

\section{ACKNOWLEDGMENT}

The authors are indebted to $\mathrm{Mr}$. Ralph Kanode for technical assistance and to Dr. Virginia McGann for helpful discussion.

\section{SOMMARY}

The influence of alterations in cultural conditions and in composition of the medium on elaboration in vitro of the protective antigen of Bacillus anthracis was investigated. Glycine, alanine, lysine, cystine, glutamine, uracil, and adenine could be omitted from the medium individually or in combination without appreciable reduction in growth or elaboration of antigen; omission of these constituents allowed considerable simplification of the method of preparation of the medium. Sodium bicarbonate, calcium ion, leucine, isoleucine, methionine, proline, phenylalanine, and histidine were critical factors in the elaboration of antigen under the experimental conditions employed. Threonine, glutamic acid, and certain purines or their derivatives apparently stimulated elaboration of antigen primarily by increasing the growth of the organism. Attempts to replace bicarbonate by certain functionally related compounds were unsuccessful. Use of a nonencapsulated, nonproteolytic mutant of $B$. anthracis was found to facilitate production of the antigen. The meaning of the results is discussed.

\section{REFERENCES}

Brawer, C. R., McCullovgr, W. G., Mills, R. C., Roessler, W. G., Herbst, E. J., AND
How , A. F. 1946 Studies on the nutritional requirements of Bacillus anthracis. Arch. Biochem., 10, 65-75.

Cromartie, W. J., Watson, D. W., Bloom, W. L., AND HeckLY, R. J. 1947 Studies on infection with Bacillus anthracis. II. Immunological and tissue damaging properties of extracts prepared from lesions of $B$. anthracis infection. J. Infectious Diseases, 80, 14-27.

Gladstone, G. P. 1946 Immunity to anthrax: protective antigen present in cell-free culture filtrates. Brit. J. Exptl. Pathol., 27, 394-418. Lankford, C. E., Swausch, C., and Ravel, J. M. 1947 Further studies on the effect of sterilizing glucose in culture media on growth on microorganisms: Utilization of cystine by lactic acid bacteria. J. Bacteriol., 63, 368.

STERNe, M. 1937 Variation in Bacillus anthracis. Onderstepoort J. Vet. Sci. and Animal Ind., 8, 271-349.

Thorne, C. B., Gomez, C. G., Bund, G. R., AND Housewright, R. D. 1953 Synthesis of glutamic acid and glutamyl polypeptide by Bacillus anthracis. III. Factors affecting peptide production in synthetic liquid media. J. Bacteriol., 65, 472-478.

Wright, G. G., ANd Stein, J. B. 1951 Studies on immunity in anthrax. I. Variation in the serum $T$-agglutinin during anthrax infection in the rabbit. J. Exptl. Med., 93, 99-106.

Wriatr, G. G., Hedberg, M. A., and Frinberg, R. J. 1951 Studies on immunity in anthrax. II. In vitro elaboration of protective antigen by non-proteolytic mutants of Bacillus anthracis. J. Exptl. Med., 93, 523-527.

Wright, G. G., Hedberg, M. A., and Slein, J. B. 1954 Studies on immunity in anthrax. III. Elaboration of protective antigen in a chemically-defined, non-protein medium. $J$. Immunol., 72, 263-269. 